\title{
Punch Rectal Biopsy in Diagnosis of Hirschsprung's Disease - An Updated Study
}

\author{
Md. Abdus Sattar ${ }^{*}$, Md. Mahfuzul Haque ${ }^{2}$, Arun Kanti Paul ${ }^{3}$, Md. Ruhul Quddus ${ }^{4}$
}

${ }^{1}$ Assistant Professor, Department of Pediatric Surgery, Pabna Medical College, Pabna, Bangladesh

${ }^{2}$ Assistant Professor, Department of Pediatric Surgery, Rangpur Medical College, Rangpur, Bangladesh

${ }^{3}$ Assistant Professor, Department of Ortho Surgery, Pabna Medical College, Pabna, Bangladesh

${ }^{4}$ Assistant Professor, Department of Biochemistry, Pabna Medical College, Pabna, Bangladesh

DOI: $10.36347 /$ sjams.2020.v08i11.016

| Received: 22.09.2020 | Accepted: 01.10.2020 | Published: 14.11.2020

*Corresponding author: Dr. Md. Abdus Sattar

Abstract

Background: Hirschsprung's disease is a common cause of neonatal \& infantile intestinal obstruction. The accurate diagnosis of HD depends on histopathological demonstration of the complete absence of ganglion cells with the presence of hypertrophied nerve fibers in the distal bowel. So, rectal biopsy is the gold standard way for diagnosis of HD. Aim of the study: This study is an attempt to find out a simple, safe, sensitive, less time consuming, cost effective with accurate histopathological results for diagnosis of HD, specially for poor and developing countries of the world. Methods: This was a cross sectional, prospective study, purposive sampling was done. Total 25 patients of suspected Hirschsprung's disease were included in this study after fulfillment of inclusion criteria from November 2010 to March 2012 at Dhaka Shishu (Children) Hospital, Dhaka, Bangladesh. Result: Out of 25 cases, 17 were diagnosed as having HD, 8 were diagnosed as having no HD. The sensitivity was $80 \%$ and positive predictive value was $94.11 \%$ of punch rectal biopsy. Operation time was only 14 minutes (on average). Cost was very less 1850 taka (on average). Post procedure hemorrhage occurred only in 1 cas, No perforation occurred among all cases. Conclusion: Punch rectal biopsy for diagnosis of HD is an advantageous method in the form of less time, less cost, less complication with efficacious.

Keywords: Hirschsprung's disease, Rectal biopsy, Ganglion Cell.

Copyright $\odot 2020$ The Author(s): This is an open-access article distributed under the terms of the Creative Commons Attribution 4.0 International License (CC BY-NC 4.0) which permits unrestricted use, distribution, and reproduction in any medium for non-commercial use provided the original author and source are credited.

\section{INTRODUCTION}

Hirschsprung's disease is a common cause of neonatal and infantile intestinal obstruction which is due to absence of ganglion cells with presence of hypertrophied nerve bundles along a variable portion of distal intestine. The incidence of HD is 1 in 5000 children with sex ratio in classic pattern male female is $4: 1$. From the beginning period of it's description the diagnosis of Hirschsprung's disease is a challenging matter to pediatritian and pediatric surgeons. Complete history taking, careful clinical examination, radiological investigation and anorectal manometry may be sufficient in many cases to suggest Hirschsprung's disease but the unique criteria for diagnosis of Hirschsprung's disease is the histopathological demonstration of the complete absence of ganglion cells with presence of hypertrophied nerve fibers in the distal bowel. Historically, rectal biopsy is the gold standard for diagnosis of Hirschsprung's disease. Upto this, there are 3 ways of rectal biopsy 1. Full-thickness rectal biopsy, 2. Rectal suction biopsy \& 3. Punch rectal biopsy. Swenson et al., in 1959 [12] first described the method of full-thickness rectal biopsy that requires admission into hospital, general anesthesia \& suturing of biopsy site. It may result in bleeding, sepsis, inflammation \& scaring which may interrupt subsequent pull through operation. Dobbin and Bill 1965 [11] and Noblett 1969 [5] introduced rectal suction biopsy for obtaining rectal mucosa and submucosa for histopathological diagnosis. It is easy to perform without anesthesia but it needs a special instrument (Great Ormond street flexible rectal suction biopsy instrument) \& there may be some notifiable complications like bleeding, perforation etc Grosfeld JL et al., 2006 [1]. Punch rectal biopsy, this is another method, first described in 1972 by Shanding \& Auldist [10]. It is also simple, safe, no need of anesthesia, done on bedside as a day case procedure with a simple biopsy forcep, or nasal cutting forcep or laryngeal biopsy forcep Hirose et al., 1993 [2] with the help of proctoscope, nasal speculum Schmitt et al., 1984 [9] or handmade biopsy tube (plastic centrifuge tube). To the best of our knowledge primary work on full-thickness, 
suction \& punch rectal biopsy have been performed in different institutions and hospitals of the world. Here, we are further interested to see the indepth of punch rectal biopsy to find out it's cost, time, complications with diagnostic accuracy as a hole.

\section{OBJECTIVES}

\section{a) General objective}

- This study is to find out a simple, safe, sensitive, less time consuming, cost effective with accurate histopathological results for diagnosis of HD.

\section{b) Specific objectives}

- Diagnosis of HD, specially for poor and developing countries of the world.

\section{Methodology \& Materials}

This was a cross-sectional, prospective study. Total 25 patients of suspected Hirschsprung's disease, age ranging from 1 day to 12 months were included in this study, after fulfillment of inclusion criteria from November 2010 to March 2012 at Bangladesh institute of child health (BICH) and Dhaka shishu (children) hospital, Dhaka. Patients with neonatal intestinal obstruction that needed immediate surgical exploration and patient's parents who were unwilling to subject their child to undergo the biopsy procedure were excluded from the study. Total 25 patients $(n=25)$ underwent punch rectal biopsy. After biopsy, all the specimens of 25 patients were evaluated by histopathological examination at pathology department of DSH. Specimen was collected from one site of rectum from each patient \& serial sections (50-60) were done from all the samples \& were stained with $\mathrm{H} \&$ E. Specimen from 2 patients was inadequate, again full-thickness rectal biopsy was performed before definitive procedure. Remaining 23 patients of punch rectal biopsy, 17 patients were diagnosed as having HD \& 6 patients were diagnosed as having no HD, again fullthickness rectal biopsy were done of this 6 patients prior to definitive procedure. We used Takahashi biopsy forcep (Fig-1) whose tip was cup likes with sharp cutting edge in both lips measuring $3 \mathrm{~mm} \times 3 \mathrm{~mm} \times 5 \mathrm{~mm}$ $\&$ a handmade biopsy tube made from plastic centrifuge tube (Fig-2) whose length was $6-8 \mathrm{~cm}$, diameter $8 \mathrm{~mm}$, side hole diameter was $8 \mathrm{~mm}$ to perform it. The rectum was cleaned with povidone iodine soaked gauze piece before biopsy \& the biopsy was performed in the lithotomy position without anesthesia.

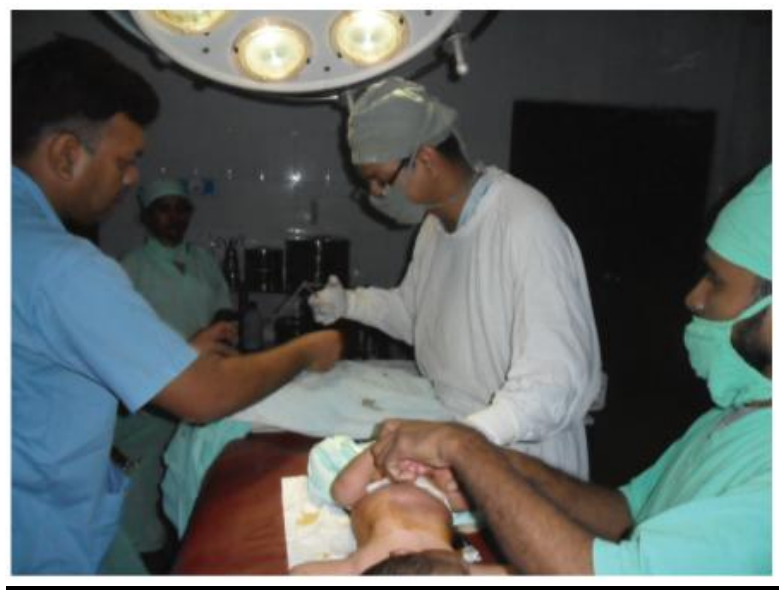

Fig-1

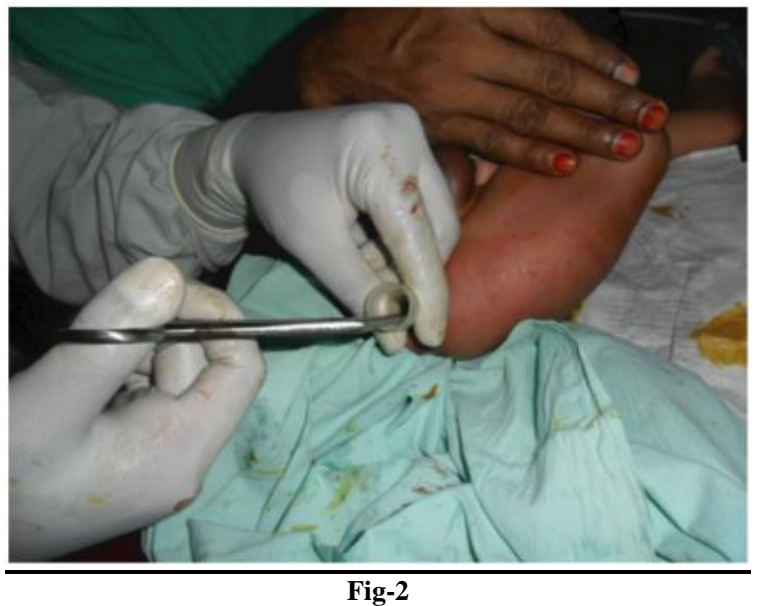

The handmade biopsy tube was inserted into the rectum with the side hole facing the posterior wall of the rectum. Slight lateral pressure was applied so that the side aperture rests firmly against the mucosal surface. The rectal mucosa protruding from the hole can be seen in the biopsy tube using a spot light as a source of light. The protruded mucosa was grasped by the biopsy forcep and pulled off (Fig-2). The edge of the side hole acts as the traction fulcrum, therefore making a small specimen of the mucosa with submucosa easily obtainable. Thereafter, the biopsy tube was turned so that pressure was applied to the biopsy site by the wall of the tube for purpose of the hemostasis. Then we kept a normal saline soaked gauze piece over the biopsy site for 3 minutes. After 3 minutes we removed the gauze piece $\&$ observed for bleeding per anus. Subsequently, we also observed features of perforation like abdominal distension, vomiting, temperature \& plain x-ray abdomen in erect posture, if needed. For the validity of the study outcome sensitivity \& positive predictive value of punch rectal biopsy was done. For significance of differences pearson test, Chi-square test, Fisher's exact test $\&$ unpaired ' $\mathrm{t}$ ' test were applied, $\mathrm{p}$ value $<.05$ was taken as statistically significant. Prior to the commencement of the study, the study protocol was approved by the ethical review committee of $\mathrm{BICH}$. The objectives of the study, the procedure, its assistance in making rational approach of the case management and 
potential complications were explained to the parents/legal guardians in easily understandable local language and then informed written consent was taken.

\section{RESUltS}

Out of 25 cases, 17 were diagnosed as having HD, inadequate sample was 2 patients, 6 patients were diagnosed as having no HD. Sensitivity and positive predictive value of punch rectal biopsy was $80 \%$ and $94.11 \%$ respectively in relation to full-thickness rectal biopsy. Time was less in punch rectal biopsy (14 minutes). Cost was less (1850 taka). Post procedure hemorrhage occurred only in 1 case but there was no perforation of any case.

Table-1: Efficacy of punch biopsy in relation to full-thickness biopsy

\begin{tabular}{|l|l|l|l|l|}
\hline \multicolumn{5}{|c|}{ (Gold standard) Full-thickness } \\
\hline & & Disease (+ve) & Disease(-ve) & \\
\hline $\begin{array}{l}\text { (Test outcome) } \\
\text { Punch }\end{array}$ & (+ve) 17 & True +ve (16) & Fasle +ve(1) & 17 \\
\cline { 2 - 5 } & $(-v e) 8$ & False -ve(4) & True -ve(4) & 8 \\
\hline Total & 25 & 20 & 5 & 25 \\
\hline
\end{tabular}

Table-2: Time, cost \& complications of biopsy procedure

\begin{tabular}{|l|l|l|}
\hline Variables & Punch rectal biopsy & P Value $(<\mathbf{0 . 0 5}$ is taken as significant $)$ \\
\hline Procedure time & & \\
\hline Range & $11-17(\mathrm{~min})$ & 0.0001 \\
\hline Mean \pm SD & $13.04 \pm 15.32$ & \\
\hline Cost & & \\
\hline Mean \pm SD & $1850.00 \pm 0.00$ & \\
\hline Complications & & \multirow{2}{*}{1.000} \\
\hline & Hemorrhage $1(4 \%)$ & \\
\hline & Perforation $0(0 \%)$ & \\
\hline
\end{tabular}

\section{DiSCUSSION}

Diagnosis of HD was definitively based on the histopathological confirmed absence of ganglion cells and the presece of hypertrophied nerve bundles. Thus, rectal biopsy which requires an appropriate \& representative tissue sample is mandatory for definitive diagnosis of HD. Full-thickness biopsy, suction biopsy $\&$ punch biopsy are currently available as rectal biopsy methods. Swenson et al., in 1959 [9] first described the method of full-thickness rectal biopsy, it is the oldest method of biopsy \& represents the tissue adequately no dought but it requires hospital admission, general anesthesia, suttering of biopsy site that may leads to bleeding, sepsis, inflammation, scaring of biopsy site \& interruption of subsequent operative procedure. In common sense, it is time consuming \& costly one. So, several attempts and works are going through the world to find out a easily performed, most accurate, less time consuming \& cheap procedure of biopsy. On this regard, Dobbin \& Bill 1965 [11] and Noblett 1969 [5] introduced rectal suction biopsy, Shandling \& Auldist 1972 [8] also introduced rectal punch biopsy. Suction \& punch biopsy are popular \& acceptable due to their low complication rates as reported by Hirose et al in 1993 [2]. At least 25 punch biopsy was performed during this study period \& the low complication rates have thus far been maintained. Our study population was 25 and among those 17 (34\%) were neonates \& 9 (18\%) were infants. Another study showed $34 \%$ neonates conducted by Islam T et al., 2004 [4] at Dhaka Shishu (Children) Hospital, Dhaka. More recent series have noted that HD is diagnosed in the newborn period in more than $90 \%$ of patients Grosfeld JL et al., 2006 [1]. One out of 5,000 children (varying 4,400 to 7,000) is born with HD with sex ratio in classic pattern male female 4:1 Russell, Russell \& Niebuhr, $1994^{6}$. In our study series, male predominates over female at a ratio of 5:2 but previous study in Dhaka shishu (Children) hospital having the ratio of 3.8:1 Alam M. I 2002 [10]. Other previous demographic study showed around the same type of results, where male female ratio 3:1 Ikeda \& Goto 1984 [3], 4.32:1 Goldberg, $1984^{12}$. So, the sex ratio of HD patient in our study was well within the expected range of different published series. In punch rectal biopsy out of 25 patients, post biopsy hemorrhage occurred in 1 case $(4 \%)$ which was insignificant and managed by wet gauze pack pressure $\&$ vit-k intravenous injection, but have no need of stitch or fresh blood transfusion. This baby was preterm \& low-birth weight $(1.5 \mathrm{~kg})$. Coagulation profile of this baby was within normal limit. We have not encountered any case of perforation. There was no others complication such as infection or abscess at biopsy site which are shown in other study Hirose et al., 1993 [2]. Yet, we feel that this type of complications can readily be avoided by proper evaluation of patients including coagulation profile before punch biopsy and more gentle approach during the procedure. Mean time was less in punch rectal biopsy (14 minutes). Cost was less (1850 Tk). Out of 25 cases of punch rectal biopsy $17(68 \%)$ were proved to be HD positive and $8(32 \%)$ to be HD negative. 2 patients had inadequate sample, these 2 cases again underwent full thickness rectal biopsy and found 1 was HD positive \& 1 was HD negative. The remaining 23 cases also underwent full-thickness rectal biopsy before 
definitive procedure to see the effectiveness of punch rectal biopsy in relation to full-thickness rectal biopsy. In Hirose et al., 1993 [2] study showed inadequate specimen was $11.2 \%$. This inadequate specimen may be due to inexpertness in obtaining tissue in early period of study.

\section{Limitations of the Study}

This was a single centered study with a small sized sample. So the findings of this study may not reflect the exact scenario of the whole country.

\section{CONCLUSION AND}

\section{RECOMMENDATIONS}

Punch rectal biopsy is a simple, safe, cost effective, less time consuming and effective procedure to diagnose the HD easily and accurately. It does not need hospitalization, pre-operative preparation, operation theater setup or general anesthesia. So, it can be taken as a first line rectal biopsy procedure obviously.

\section{REFERENCES}

1. Grosfeld JL. HD \& related neuromuscular disorder of intestine" pediatric surgery, St Lous: Mosby, $6^{\text {th }}$ eds. Philadelphia, USA: ELSEVIER, 2006;15141514.

2. Hirose R, Hirata Y, Yamada T, Kawana T, Taguchi $\mathrm{T}$, Suita $\mathrm{S}$. The simple technique of rectal mucosal biopsy for the diagnosis of Hirschsprung's disease. Journal of pediatric surgery. 1993 Jul 1;28(7):9424.

3. Ikeda KE, Goto SE. Diagnosis and treatment of Hirschsprung's disease in Japan. An analysis of
1628 patients. Annals of surgery. 1984 Apr;199(4):400-405.

4. Islam T. Rectal punch biopsy in Hirschsprung's Disease, MS thesis, BICH, University of Dhaka, Dhaka. 2004.

5. Noblett HR. A rectal suction biopsy tube for use in the diagnosis of Hirschsprung's disease. Journal of pediatric surgery. 1969 Aug 1;4(4):406-9.

6. Russell MB, Russell CA, Niebuhr E. An epidemiological study of Hirschsprung's disease and additional anomalies. Acta Paediatrica. 1994 Jan;83(1):68-71.

7. Schmitt M, Plenat F, Pierre E, Vignaud JM. Biopsy of the rectal mucosa in pediatric surgery. Technic-diagnostic value. Apropos of 193 biopsies. Chirurgie Pediatrique. 1984 Jan 1;25(2):98-101.

8. Shandling B, Auldist AW. Punch biopsy of the rectum for the diagnosis of Hirschsprung's disease. Journal of pediatric surgery. 1972 Oct 1;7(5):54652.

9. Swenson O, Fisher JH, Gherardi GJ. Rectal biopsy in the diagnosis of Hirschsprung's disease. Surgery, 1959;45(113):690-695.

10. Alam MI. Role of immuniohistochemical stain in rectal suction biopsy specimen for the diagnosis of Hirschsprung's" MS Thesis, BICH, University of Dhaka, Dhaka. 2002.

11. Dobbins III WO, Bill Jr AH. Diagnosis of Hirschsprung's disease excluded by rectal suction biopsy. New England Journal of Medicine. 1965 May 13;272(19):990-3.

12. Goldberg EL. An epidemiological study of Hirschsprung's disease. International journal of epidemiology. 1984 Dec 1;13(4):479-85. 